\title{
A research on influencing factors of lottery buyer fatigue
}

\author{
Shan Liang ${ }^{1, a}$
}

${ }^{1}$ Department of Q-Economics, School of Economics and Commerce, South China University of Technology, Guangzhou, China

a1057432985@qq.com

*Shan Liang

Keywords: lottery buyer fatigue, lottery behavior, correlation analysis.

\begin{abstract}
This paper puts forward the concept of lottery buyer fatigue on the basis of gamblers fatigue, and studies the phenomenon that lottery holders produce adaptability - adjusting the behavior of purchasing in the process of buying lotteries. Through correlation analysis and comparative analysis, this paper summarizes the characteristics of lottery buyer fatigue group including women, 18 to 30 years old, more than a high school level of education. At the same time, this study points out that the social environment has great correlation with lottery buyer fatigue phenomenon, while the correlation between family environment and lottery buyer fatigue phenomenon is smaller.
\end{abstract}

\author{
彩民疲劳影响因素研究 \\ 梁珊 $1, a$ \\ 1华南理工大学,经济与贸易学院, 广州, 广东, 中国 \\ a1057432985@qq.com \\ 梁珊
}

关键词：彩民疲劳; 购彩行为; 相关分析

中文摘要. 本文在奢客疲劳的基础上提出了彩民疲劳概念, 对彩民购彩过程中产生适应性, 调整购彩行为甚至停止购彩的现象进行研究。通过相关分析及对疲劳彩民和潜在问题彩 民与各影响因素的相关关系大小的对比分析，总结出产生彩民疲劳现象的彩民群体特征 包括女性、18-30 岁青年、高中以上受教育程度等。同时指出社会环境与彩民疲劳现象 有较大相关性，而家庭环境与彩民疲劳现象的相关性则较小。

\section{1. 引言}

近年来来, 我国彩票业迅猛发展, 彩民的数量迅猛增加。国内学者研究主要集中于问题 彩民的行为及其影响因素, 主要成果有: 史文文、王斌（2013）总结了四个影响购彩成瘾的 因素，即个体因素、家庭经济状况、不良信息的传播、经济效用; 刘炼(2012)发现外部信息 作用对彩民的影响比较大。而与问题彩民对立的则是疲劳彩民，疲劳彩民是指彩民对彩票产 生适应性，即彩民购彩一段时间之后，调整购彩行为，降低购彩频率、购彩金额等，甚至停 止购彩，并把这种现象为 “彩民疲劳”，该概念基于曾忠禄 “奢客疲劳” 概念提出，曾忠禄 （2006）提出奢客疲劳一词，认为奢客疲劳是指奢客对奢博的适应性，奢客调整自己的购彩 行为甚至停止奢博的现象。Brian J. Zikmund-Fisher（2002）通过实验研究了为什么人们会继 
续或放弃一项连续的行动, 并提出了三个原因: 积极的近期预测、沉没成本的心理预算和累 计概率评估。DAVID C. HODGINS等 (2010) 指出博彩者停止博彩行为受到四个因素的影响, 分别是经济状况、家庭因素、对痛苦、压力的忍受程度、个人的行为方式。

综上所述，研究内容上，国内现有研究主要集中在问题彩民及其影响因素的研究，鲜有 关于彩民疲劳的文献, 国外则已经有对 “适应性” 的研究成果, 而研究方法上, 以定性研究 方法居多。本文提出了彩民疲劳的概念, 弥补了博彩业关于彩民产生适应性现象的理论空白, 研究方法上, 通过相关分析和对比分析方法, 定量分析产生彩民疲劳现象的群体特征及关键 因素。

\section{2. 分析方法介绍}

\section{1 研究对象}

彩民疲劳是指彩民连续购彩一段时间后，对彩票产生适应性，从而调整自己的购彩行为 的现象。由于适应性的产生需要一段时间, 所以本文的研究对象为持续购买彩票半年以上的 彩民。

本次问卷调查面向广州市民共发放问卷120份，收回有效问卷108份，回收率90\%，对回 收数据进行审核, 删除有逻辑错误、填写错误的问卷，实际有效问卷为 101 份，其中 9 名受访 者的购彩时长不足半年，因此实际研究所用调查问卷为92份。

\section{2 彩民疲劳界定方法}

对疲劳彩民界定和测量的准确性及有效性是研究问题的关键。借鉴问题彩民判断标准的 两种方法 (是否回答的强制判断量表方法和等级判断的Likert式量表方法) 可知, 对彩民疲劳 的界定, 首先需要明确购彩行为调整程度, 然后划定阈值, 做出判定。具体方法如下:

（1）考察购彩行为调整的程度。考察购彩行为调整的维度包括购彩动机、花费时间、单 次购彩金额、购彩频率、是否研究中奖规律、购彩资金是否合理安排等六个维度，每个维度 20 分, 若该维度为正向调整记20分，若为负向调整则记-20分。这里的正向调整具体指从初期 到现期，彩民花费时间减少、购彩金额、购彩频率降低，购彩动机由中大奖向娱乐、奉献爱 心转变，从初期研究中奖规律到现期不再研究中奖规律，从初期购彩金额安排不合理到现期 合理安排; 反之则为负向调整。

（2）划定阈值。根据打分原则，计算得分，得分50分之上的彩民判定为 “疲劳彩民”; 得分在- 80 以下的彩民判定为 “潜在问题彩民”。在本次调查中包含疲劳彩民 6 人，占到总样 本的 $6.52 \%$; 包含潜在问题彩民 9 人, 占总样本的 $9.78 \%$ 。

\section{3 变量解释}

研究文献，总结学者对问题彩民的影响因素研究成果，发现其影响因素可以总结为个人 因素和环境因素。个人因素包括性别、年龄、受教育程度、收入、彩民心理特质及彩票认知， 其中彩民心理特质与彩票认知的量化通过打分方法进行; 环境因素包括家庭环境和社会环境。 家庭环境主要指家人对彩民的劝告与管教等, 社会环境指彩民周围朋友对彩票的谈论程度与 彩民朋友对彩票的痴迷程度等, 具体标准如表1:

表1 心理特质及彩票认知打分原则

\begin{tabular}{|c|c|c|c|}
\hline 维度 & 评价内容 & 是 & 否 \\
\hline \multirow{5}{*}{ 心理特质 } & 是否有不同业余爱好 & 20 & -20 \\
& 欠债是否会感到不安 & 20 & -20 \\
& 与朋友的聚会能否都尽情玩 & 20 & -20 \\
& 日常生活是否乱扔废纸、垃圾 & -20 & 20 \\
& 难堪经历会长时间难受否 & -20 & 20 \\
\hline
\end{tabular}




\begin{tabular}{|l|c|c|c|}
\hline \multirow{5}{*}{ 彩票认知 } & 中奖号码选择是否有技巧 & -20 & 20 \\
& 智力对中奖是否有影响 & -20 & 20 \\
& 彩票中奖规律是否能研究出来 & -20 & 20 \\
& 彩票中奖概率是否小于千分之一 & -20 & 20 \\
& 是否能区分不同彩种 & 20 & -20 \\
\hline
\end{tabular}

\section{4 相关系数计算}

所有的影响因素中包括两类变量, 定性变量和定量变量。对于定量变量, 相关系数的计 算采用皮尔逊相关系数, 而针对定性变量, 其相关系数的计算按照如下定义进行:

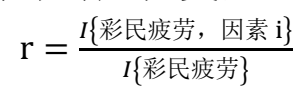

其中, I $\{$ 彩民疲劳 $\}$ 表示满足彩民疲劳条件的彩民数, I\{彩民疲劳, 因素 $\mathrm{i}\}$ 表示同时满足彩 民疲劳条件与存在因素 $\mathrm{i}$ 的条件的彩民数, 相关系数 $\mathrm{r}$ 的取值在 $[0,1], \mathrm{r}$ 越大表示因素 $\mathrm{i}$ 与彩民疲 劳现象的相关关系越大。

\section{3. 数据分析}

性别、年龄、受教育程度、家庭环境、社会环境按照定性变量相关系数计算方法计算, 收入、心理特质及彩票认知采用皮尔逊相关系数进行计算, 彩民疲劳现象与各因素的相关系 数计算结果见表 4 中 $\gamma_{1}$ 。由于 “彩民疲劳” 与 “彩民成瘾” 是两种在彩民中共存的现象, 将潜 在问题彩民与疲劳彩民进行对比分析, 进一步明确产生彩民疲劳现象的关键因素, 采用同样 的相关系数计算方法计算, 潜在问题彩民与各因素的相关系数计算结果见表 3 中 $\gamma_{2}$ 。

表3 彩民疲劳影响因素相关系数

\begin{tabular}{|l|c|c|c|}
\hline & 影响因素 & 相关系数 $\gamma_{1}$ & 相关系数 $\gamma_{2}$ \\
\hline \multirow{5}{*}{ 个人因素 } & 性别 (女) & 0.69 & 0.44 \\
& 年龄 (18-30) & 0.67 & 0.27 \\
& 受教育程度 (高中以上历) & 1 & 0.11 \\
& 收入 & 0.78 & -0.86 \\
& 心理特质 & 0.37 & -0.40 \\
& 彩票认知 & 0.43 & -0.45 \\
\hline \multirow{2}{*}{ 环境因素 } & 家庭环境 & 0.17 & 0.89 \\
& 社会环境 & 0.67 & 0.78 \\
\hline
\end{tabular}

单独分析 $\gamma_{1}$ 和 $\gamma_{2}$ 。由相关系数 $\gamma_{1}$ 可知, 性别、年龄、受教育程度、收入、社会环境与彩 民疲劳现象的相关关系较大, 家庭环境与彩民疲劳现象不相关。由相关系数 $\gamma_{2}$ 可知, 收入、 家庭环境、社会环境与潜在问题彩民有较强相关性。

对比分析发现:

（1）女性却更容易产生适应性，向疲劳彩民方向发展; 而男性则相反。

（2）疲劳彩民和潜在问题彩民在年龄、受教育程度和收入上具有显著差异; 年龄上, 疲 劳彩民集中于18-30岁之间, 潜在问题彩民则在30岁以上; 学历及收入上, 疲劳彩民多为高中 以上学历且为中高收入人群，而潜在问题彩民更多为高中及以下学历，低收入人群。

（3）家庭环境与潜在问题彩民的相关关系相对较大，而与疲劳彩民则几乎不相关。分析 原因可能为家人对彩民的劝告与管教往往发生在彩民购彩行为影响到正常生活之后, 其滞后 性导致家庭环境对彩民适应性的产生无促进作用，相反会引起家庭矛盾。

（4）心理特质和彩票的认知对彩民疲劳和潜在问题彩民的影响方向相反，但影响程度均 不大。结果表明, 大部分彩民均对彩票有正确认知且有健康的心理, 其购买彩票的目的已经 不是仅仅为了赢钱，而是获得购彩带来的快乐感。

（5）社会环境因素中，周围彩民朋友谈论彩票、对彩票痴迷与彩民疲劳和彩民成瘾现象 均有较大相关关系。可能是受到彩民受教育程度的影响, 疲劳彩民的受教育程度更高, 是非 
辨别能力越强, 能从周边人的言论及行为中吸取教训; 而潜在问题彩民更容易受到周围人的 影响，逐渐偏离正确轨道，发展为问题彩民。

\section{4. 结论及建议}

本研究提出了 “彩民疲劳” 概念，填补了彩民博彩现象的理论空白，并从彩民疲劳现象 影响因素出发提出预防病态博彩的合理建议。

（1）个人因素方面，性别、年龄、受教育程度及收入对适应性的产生均有显著影响。容 易产生适应性的彩民群体主要特征有：性别女性、年龄18-30岁、较高学历及收入。因此对于 彩民而言，应该提高自身的知识水平，合理分配时间，对外部环境信息要认真辨别，去伪存 真;

（2）环境因素方面，社会环境对彩民有重要影响，而家庭环境影响较小。但是对于彩民 家人朋友来说，应当尽早提醒彩民购彩可能存在的风险以及危害，以免误入歧途; 对于彩民 来说应该慎重交友, 同时对夸大彩票收益的行为进行劝告, 并提高自己的防范意识; 对于政 府来说，应该履行职能，在彩票宣传过程中提醒彩民健康博彩，预防病态博彩。

\section{References}

[1] Wang Bin and Shi wen-wen. The influential factors and mechanism of purchasing lottery addiction of sports lottery players. China Sport Science And Technology. vol. 49, pp. 130-136, 2013.

[2] Liu Lian. Relations of Purchase Intention and Behavior in Sports Lottery Consumers in China[D].Wuhan, Central China Normal University, 2012.

[3] Zeng Zhonglu, Zhai Qun and You Xuqun. A study on the bounded rationality of lottery buyers in China. Psychological Science. vol. 32, pp.1248-1251, 2009.

[4] Brian Joseph and Zikmud-Fisher. Continue or Quit Decisions in Sequential Trial Situations[D]. Pittsburgh, Carnegie Mellon University, 2002.

[5] David C. Hodgins and Nady el-Guebaly, The influence of substance dependence and mood disorders on outcome from pathological gambling: five-year follow-up. Journal of Gambling Studies. vol. 26, pp. 117-127, 2010.

[6] Randy Stinchfield, Reliability, Validity, and classification accuracy of the South Oaks Gambling Screen (SOGS);Addictive Behaviors. vol. 27, pp.1-19, 2002. 